\title{
Breast Cancer MDCs (Multidisciplinary Clinics) and Related Ethical Issues ${ }^{1}$
}

\author{
Cliniques multidisciplinaires pour le cancer du sein et les problèmes éthiques liés
}

\author{
E. Murray • E. Panieri \\ (C) Springer-Verlag France 2013
}

\begin{abstract}
Although often regarded as a cancer of the developed world, breast cancer presents a serious problem to poorer countries. Large populations, late presentation and poor access to medical care mean that mortality rates are relatively high and a large number of total deaths occur [1]. In situations where resources are scarce, the application of evidence-based medicine is essential when deciding how to use what resources are available. In many countries, particularly in the developed world, it is considered standard practice to manage breast cancer patients via a multidisciplinary clinic [2]. A Breast Cancer Multidisciplinary Clinic (MDC) or Tumour Board has many potential benefits for both practitioners and patients, and we feel represents a standard of practice that is appropriate for all countries.

A Breast Cancer MDC should be attended by at least one surgeon and one oncologist and, ideally, a radiologist as well as a pathologist. A plastic surgeon, a breast nurse, a social worker or psychologist and a geneticist or genetics counselor are also frequently involved. Recommendations as to its structure and functioning have been made by various bodies and detailed NICE guidelines are readily available [3]. There is a positive association between the proportion of breast cancer nurses in the team and the overall measure of clinical performance [4]. The establishment of criteria to be met by the clinics is of paramount importance. Every meeting of doctors caring for breast cancer patients may not fulfil the usual criteria for an MDC and it would be erroneous to
\end{abstract}

E. Murray $(\triangle) \cdot$ E. Panieri

Radiation oncologist, private practice, Cap Town, South Africa

e-mail : elizabeth.murray@telkomsa.net

\footnotetext{
${ }^{1}$ Dr Elizabeth Murray (Oncology) and Professor Eugenio Panieri (Surgery) co-chaired the Breast Cancer MDC at Groote Schuur Hospital, Cape Town, South Africa, for many years. Dr Murray is currently in private practice, where she has founded an independent MDC, while Prof Panieri remains the head of the Groote Schuur Hospital Breast Clinic. They are both members of the International Breast Cancer Study Group and the Early Breast Cancer Trialists' Collaborative Group
}

assume that the benefits would be the same if criteria are not met. A clinic that is not based on sound ethical principles may be worse than useless, confirming non-evidence-based 'usual practice' rather than ensuring the outcomes' benefits of evidence-based medicine. It is however difficult to prescribe on functional aspects and group dynamics.

It is useful to spell out the principles on which an MDC is founded in an introductory letter for new members. Issues included may be the use of evidence-based medicine (not as obvious a principle to some as to others!), how decisions are to be used (in the setting of independent clinicians it may be wise to reassure them that legally and ethically the recommendation to their patient will always be theirs) and who will be welcomed as participants, including required qualifications, registration, experience and requisite time per week spent on breast cancer cases, for example as set out in the NICE guidelines [3]. In some under-resourced African countries, lack of availability of adequately qualified specialists may lead to less rigid or different requirements. The option of tele-conferencing where expertise is lacking can also be explored.

MDCs are not a replacement for educational meetings, although educational input forms part of the discussion and an MDC does provide a time where role-modelling for trainees can take place, teaching all participants to treat patients according to evidence-based guidelines and with care and respect, whether in their absence or presence.

A well-run MDC creates a collegial atmosphere in which evidence-based practices and the use of guidelines can be reinforced and options of investigation and treatment considered. A wide variety of treatments can be made available to patients and patient confidence is improved if the concept of the clinic is explained to them. A well-run clinic can act as a safety check ensuring the participants have peer review and are performing to the best of their ability. In addition, in under- resourced countries a well-functioning MDC team may be more successful in motivating for resources than individual doctors and the team may develop 'twinning' or research relationships $[5,6]$ with teams from developed countries. 
Although many potential benefits are recorded, improvement in outcomes from the use of MDCs in cancer in general is not a consistent finding [2,7-10]; however, this does not detract from the many advantages that this forum of clinical activity engenders.

Although an MDC may provide support to members and build morale, it should not be viewed primarily as a support for health care workers and nor is it a 'back up' in case of patient complaint. Other recorded difficulties include political rivalries and conflicting economic needs thwarting the formation of an MDC [11]. Ideally time spent should be reimbursed but we have not found that a voluntary unpaid clinic prevents participation. We do not agree with several authors who state that MDCs are an expensive entity $[9,10]$, and in real terms we have not found this to be the case in our setting.

In Cape Town, South Africa, Breast Cancer MDCs take place in the public and private sectors. At Groote Schuur Hospital, one of the main teaching hospitals in the public sector, a 'Combined Breast Clinic' with formal protocols has been running since the 1950s. In this setting patients are interviewed, although by a limited number of team members so they do not feel intimidated. Their fitness for therapy can be assessed and their preferences taken into account as the discussion with them evolves. A final treatment recommendation can be made to the patient in the clinic. This is always by a member of the surgical team if the recommendation is surgical or of the oncology team if the primary recommendation is for systemic or radiotherapy treatment, and the ultimate responsibility lies with the senior doctor in that discipline. In the private sector patients are discussed but not seen at the MDC, and the treating doctor remains responsible for the final recommendation. It is not uncommon, and indeed it may be a healthy dynamic, for panel members to disagree on certain aspects of management where options are available or evidence is lacking (i.e. evidence-based approach is not available), but this requires maturity and collegiality on the part of participants.

The responsibility for the final treatment recommendation may differ according to the type of clinic [7] and the law of a particular country, but in South Africa this is always the duty of the treating doctor, and cannot be devolved onto a committee. In the case of a complaint, however, committee members or anyone who influences the decisions could share liability, so team members do need to take care not to be swayed by external factors or other influences [12].

Confidentiality for patients and clinicians must be considered. In South Africa, in the private sector, we need consent (verbal or part of a signed registration form) from patients before discussing them with other clinicians. In the public sector the whole team is regarded as the patient's caregiver. We also have a principle that names of doctors not attending the clinic will not be discussed if, for example, members of the MDC disagree with their past or proposed future management of the patient.

Leadership of a multidisciplinary breast clinic is traditionally the role of the surgeon, but can be shared or rotated among different disciplines. Care must be taken that the treatment recommendations are those of the relevant experts and not just the clinic chair. We believe that a participatory style of leadership is essential. An overall chair is required, but a good leader will provide a forum where opinions are freely voiced rather than members deferring to a senior doctor or a doctor who is a source of referrals for them. At all times patient interests should be paramount.

Sound professional and ethical guidelines would include the keeping of records and communication with the patient's family doctor and other referring physicians.

The question of whether tumour boards or MDCs are truly worthwhile has recently been mooted $[2,13]$, but we would suggest that the issue is really how to ensure that an MDC functions optimally, in order to ensure the best possible patient outcomes and the optimal use of available resources and skills. Such an environment is often the catalyst for writing evidence-based protocols and the group can be very powerful when lobbying for much-needed resources.

\section{References}

1. Forbes JF (1997) The incidence of breast cancer: the global burden. Public health considerations. Semin Oncol 24:S1-S35

2. Keating NL, Landrum MB, Lamont EB, et al (2012) Tumor boards and the quality of cancer care. JNCI. DOI: $10.1093 / \mathrm{nci} / \mathrm{djs} 502$

3. NICE multidisciplinary team. www.nice.org.uk/guidance

4. Amir Z, Scully J, Borrill C (2004) The professional role of breast cancer nurses in multi-disciplinary breast cancer care teams. Eur Oncol Nurs Soc 8:306-14

5. Tognoni G (1993) North-south asymmetry. Ann Oncol 4:7-8

6. Murray EM (2003) Medical and radiation oncology for breast cancer in developing countries with particular reference to locally advanced breast cancer. World J Surg 27:924-7

7. Rusthaller T, Roe H, Thurliman B, Nicoll JJ (2006) The multidisciplinary meeting: an indispensable aid to communication between different specialities. Eur J Cancer 42:2459-62

8. Chirgwin J, Craike M, Gray C, et al (2010) Does multidisciplinary care enhance the management of advanced breast cancer?: evaluation of advanced breast cancer multidisciplinary team meetings. J Oncol Pract 6(6):294-300

9. Fleissig A, Jenkins V, Catt S, Fallofield L (2006) Multidisciplinary teams in cancer care: are they effective in the UK? Lancet Oncol 7:935-43

10. Saini KS, Taylot C, Ramirez A-J, et al (2012) Role of the multidisciplinary team in breast cancer management: results from a large international survey involving 39 countries. Ann Oncol 23 (4):853-9

11. Vinod SK, Sidhom MA, Delaney GP (2010) Do multidisciplinary meetings follow guideline-based care? J Oncol Pract 6(6):276-81

12. Personal communication. Elsabe Klinck Health Sector Consultant

13. Blayney DW (2012) Tumour boards (team huddles) aren't enough to reach the goal. JNCI Editorial. DOI: 10.1093/jnci/djs523 